
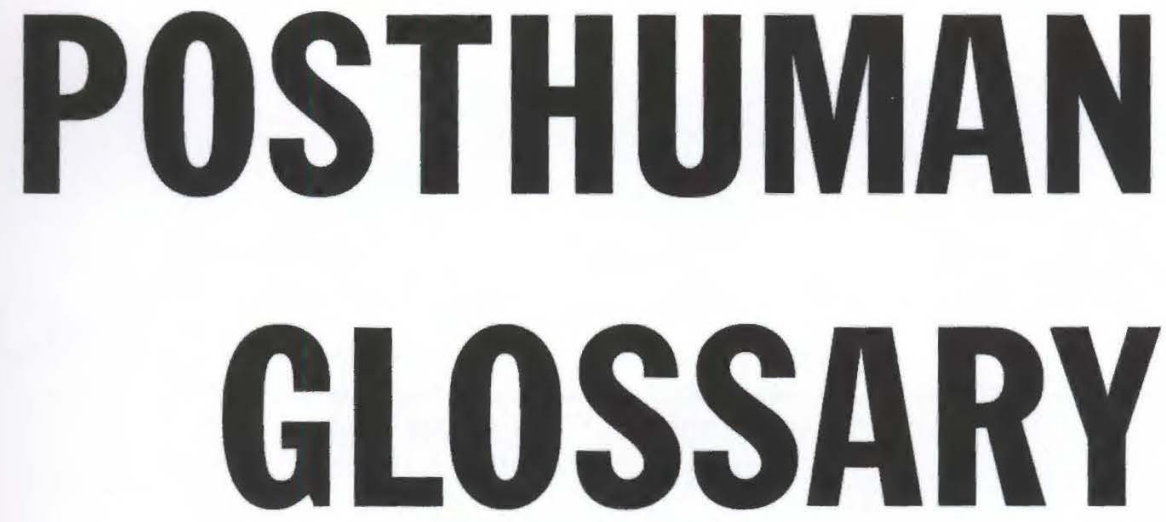

Edited by Rosi Braidotti and Maria Hlavajova

\title{
BLOOMSBURY ACADEMIC
}

An imprint of Bloomsbury Publishing Plc

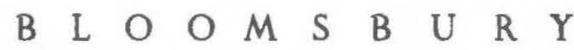

LONDON - OXFORD - NEW YORK - NEW DELHI - SYDNEY 


\section{Contents}

Acknowledgements

Series Preface

List of Contributors xii Altergorithm

xiii Timotheus Vermeulen

xiv

Animacies

MelY. Chen

1 Animal

Oxana Timofeeva

Animism (Limulus)

Karen Kramer

15

Affective Turn

Heather Houser

Afrofuturism

Ramon Amaro

Ahuman, the

Patricia MacCormack

Al (Artificial Intelligence)

Luciana Parisi

Algorithm

Jamie 'Skye' Bianco

Algorithmic Studies

David Theo Goldberg and Jenna Ng

Alienation

James Williams
Animism

Anselm Franke

17

Anonymity

41

Matthew Fuller

20

Anthropism/Immanent Humanism

44

Neni Panourgiá

21

Anthropocene Observatory

45

John Palmesino and Aun-Sofí

23 Rönnskog - Territorial Agency and

Armin Linke

26 Anthropocene

51

Jussi Parikka

28 Anthrōpos

53

\section{9}

1 
Architectonic Disposition

Vera Bühimann

Art

Tom Holert

Art in the Anthropocene

Heather Davis

\section{B}

Biological Arts/Living Arts

Oron Catts

Bios

Neni Panourgiá

Blue Humanities

Steve Mentz

Bodies Politic

John Protevi

Body Without Organs

Patricia Pisters

\section{C}

Camp

Ethel Brooks

Capitalocene and Chthulucene

Donna Haraway

Commons, the

Lindsay Grace Weber

Commutation Ontology

Lila Athanasiadou

Computational Turn

Luciana Parisi

Contemporary, The

Tom Holert
55 Cosmopolitics

Maja and Reuben Fowkes

59 Critical Posthumanism

Stefan Herbrechter

63

\section{D}

Decolonial Critique

Shannon Winnubst

66 Diffraction

99

Iris van der Tuin

68 Digital Citizenship

101

Bernhard Rieder

69 Digital Philosophy

104

M. Beatrice Fazi

72 Digital Rubbish

107

Jennifer Gabrys

74

\section{E}

Earth

110

Jussi Parikka

77 (Material) Ecocriticism

112

Serenella lovino

79 Ecohorror

115

Christy Tidwell

83 Ecologies of Architecture

117

Andrej Radman

86 Ecomaterialism

120

Serpil Oppermann

88 Econtology

123

Henk Oosterling

91 Ecopathy

125 
Ecosophy

Rick Dolphijn

Epigenetic Landscape

Susan M. Squier

Equation (Mathematical

Thinking)

Vera BühImann

Ethereal Scent

Wander Eikelboom

Exclusion Zone

Trevor Paglen

Execution

Critical Software Thing

Expulsions

Saskia Sassen

Extended Cognition

Goda Klumbytè

Extinction

150

Claire Colebrook

\section{F}

Feminicity

154

Felicity Colman

Feminist Posthumanities

Cecilia Äsberg

Food

160

Karl Steel

Forests

162

Paulo Tavares

Four Elements

Gary Genosko

129

132

167
Gaga Feminism

170

Jack Halberstam

General Ecology

172

Erich Hörl

Geo-hydro-solar-bio-techno-politics

175

John Protevi

Geomythologies

178

The Otolith Group

Geopolitics

181

Ryan Bishop

Green/Environmental Humanities

184

Tobijn de Graauw and Elisa Fiore

Gulf Labor

MTL Collective (Nitasha Dhillon and Amin Husain)

\section{$\mathrm{H}$}

Hacking Habitat

191

Ine Gevers

Hypersea

193

Jenna Sutela

Hypersocial

195

157 Tiziana Terranova

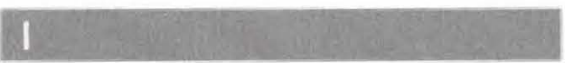

Informatic Opacity

198

Zach Blas

In-human, The

199

Katerina Kolozova

In/Human

201

Keti Chukhrov 
Insurgent Posthumanism

Dimitris Papadopoulos

Interest/Interesse

Henk Oosterling

Intermediality

Henk Oosterling

Imvariance

Vera Bühlmann

'It'

Stuart McLean

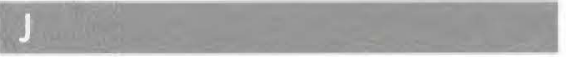

Joy, Ethics of

221 Timotheus Vermeulen

Rosi Braidotti

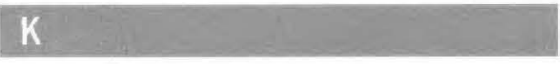

Kin

225

Goda Klumbytè

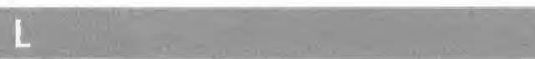

Lampedusa

228

Sandro Mezzadra and Brett Neilson

Leaks and Stings

Ravi Sundaram

Literature of Liberation

Serenella lovino

Locality/Non-separability

Lila Athanasiadou

\section{M}

MakeHuman

Femke Snelting and Jara Rocha
204 Material Feminisms

242

Astrida Neimanis

244

Brandon Jones

210 Maxwell's Demon

(Non-Anthropocentric Cognition)

Vera BühImann

Medianatures

251

Jussi Parikka

Metadata Society

253

Matteo Pasquinelli

Metamodernism

256

Metastability

Aud Sissel Hoel

Monster/The Unhuman

261

Nikita Mazurov

Multispecies

Eben Kirksey

Multiverse

Francesca Ferrando

Naturecultures

Iris van der Tuin

Necropolitics

235

Christine Quinan

Negentropy

Vera BühImann

Neo/New Materialism

277 
Neocolonial

Sandra Ponzanesi

Neocybernetics

Bruce Clarke

Networked Affect

Susanna Paasonen

Neuronal Aesthetics

Patricia Pisters

Noise

Inigo Wilkins

Nomadic Sensibility

Daniel Baker

Non-human Agency

Jeffrey Scott Marchand

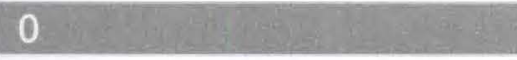

Object-oriented Ontology (000)

Peter Wolfendale

Obsolete Technologies

Tamara Shepherd and Koen Leurs

Occupy (after Deleuze)

Rick Dolphijn

Ontological Turn, the

Jeffrey Jerome Cohen

Organization in Platform Capitalism

Geert Lovink and Ned Rossiter

Otherwise Embodied Others

Pierre Huyghe

$$
\text { P }
$$

P2P (Peer to Peer) Economies

299
279 Pill, the (Posthuman Icon)

312

Anneke Smelik and Elisa Fiore

281 Placenta Politics

315

Rosi Braidotti

283 Planetary

318

Maja and Reuben Fowkes

285 Plasticity

Tom Giesbers

287 Political Affect

John Protevi

290 Post internet

326

Tom Clark

292 Postanimalism

329

Tsz Man Chan

Postdisciplinarity

332

Nina Lykke

Postglacial

335

Ursula Biemann

Posthuman Critical Theory

339

Rosi Braidotti

301 Posthuman Disability and DisHuman

Studies

304 Dan Goodley, Rebecca Lawthom, Kirsty Liddiard and

Katherine Runswick-Cole

Posthuman Ethics

345

Patricia MacCormack

Posthuman Literature and

Criticism

Carolyn Lau

Posthuman Museum Practices

Fiona R. Cameron

Michel Bauwens and Vasilis Kostakis 
Posthuman Rights, A Micropolitics of

Patrick Hanafin

Posthuman Sexuality

Patricia MacCormack

Posthumanism

Cary Wolfe

Posthumanist Performativity

Elisa Fiore

Postimage

Ingrid Hoelzl

Postmedieval

Eileen A. Joy

Precognition

Ramon Amaro

Pregnant Posthuman, The

Rodante van der Waal

Process Ontologies

James Williams

\section{Q}

Quantum Anthropology

374

Vicki Kirby

\section{R}

Radical Mediocrity

Henk Oosterling

Rationalist Inhumanism

Peter Wolfendale

Real Cool Ethics

Shannon Winnubst

Resilience

356

359
Rewilding

Maja and Reuben Fowkes

355 Robophilosophy

Johanna Seibt

Sensing Practices

394

Jennifer Gabrys and Helen Pritchard

Socially Just Pedagogies

396

Vivienne Bozalek

Speculative Posthumanism

398

David Roden

SS $=$ Security/Surveillance

402

Stephanie Simon

Stateless State

404

Jonas Staal

Static Glow

Mirko Tobias Schäfer and

Audrey Samson

Storied Matter

411

Serpil Oppermann

Survival

Alice Creischer and Andreas Siekmann

Symbiogenesis

Bruce Clarke

$$
T
$$

Technicity

420

Aud Sissel Hoel

Technoanimalism

423

Jeremy Walker and Melinda Cooper 
Terrestrial

Birgit M. Kaiser and Kathrin Thiele

Tolerances and Duration

Harry Sanderson and

Alexandra Symons Sutcliffe

Trans*

Goda Klumbyté

Trans-corporeality

Stacy Alaimo

Transhumanism/Posthumanism

Francesca Ferrando

\section{U}

(Un)Documented Citizenship

Ernst van den Hemel

Urbanibalism

442

Wietske Maas and Matteo Pasquinelli

$$
\text { V }
$$

Vertigo Sea

John Akomfrah

Vibrant Matter

Jane Bennett

427

429

433

435

438

445
Violence

Shela Sheikh

$$
\text { W }
$$

War

453

Jolle Demmers

Wearable Technology

Anneke Smelik

\section{$x$}

Xenofeminism

459

Helen Hester

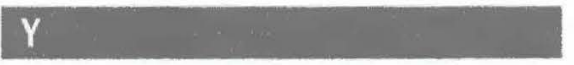

Youth

463

Koen Leurs, Tamara Shepherd and Alison Harvey

\section{Z}

Zombie

467

Oxana Timofeeva

447 Cumulative Bibliography

470 